\title{
System Providing Discomfort Monitoring for People in Wheelchairs
}

\author{
J.G. Rocha ${ }^{1}$, H. Carvalho ${ }^{2}$, F.M. Duarte ${ }^{3}$, M. A. F. Carvalho ${ }^{4}$, V.M. Moreira ${ }^{5}$ \\ ${ }^{1}$ Dep. Industrial Electronics, University of Minho, Campus de Azurem, 4800-058 Guimaraes, Portugal. \\ ${ }^{2,4}$ Dep. Textile Engineering, University of Minho, Campus de Azurem, 4800-058 Guimaraes, Portugal. \\ ${ }^{3}$ Dep. Polymer Engineering, University of Minho, Campus de Azurém, 4800-058 Guimarães, Portugal. \\ ${ }^{5}$ Sao Marcos Hospital, Largo Carlos Amarante, 4701-965 Braga Portugal.
}

${ }^{1}$ gerardo@dei.uminho.pt, ${ }^{2}$ helder@det.uminho.pt, ${ }^{3}$ fduarte@dep.uminho.pt, ${ }^{4}$ migcar@det.uminho.pt, ${ }^{5}$ moreiravitor@netcabo.pt

\begin{abstract}
This article describes a project that aims to develop a system that provides people in wheelchair a relief to the level of the sensitive perception of discomfort, assuring greater independence, welfare, quality of life and the prevention of illnesses/wounds. The project is based on the development of textile and polymer applications (cushions, mattresses and mattresses overlays) with functions of pressure and humidity
\end{abstract}

\section{INTRODUCTION}

People with serious motor limitations have, in most of the cases, a decrease of sensitivity in the body's areas in contact with the support surfaces. Their limited motor capacity does not allow them to regularly change position autonomously, as a healthy person would do unconsciously. Consequently, insufficient sanguineous irrigation occurs as result of pressure exceeding the tissue capillary pressure for a long time, depriving tissues of oxygen and essential nutrients, owing to ischemia and hypoxia, which then causes the development of pressure ulcers. Figure 1 shows the evolution to a serious pressure ulcer with the bone being exposed.
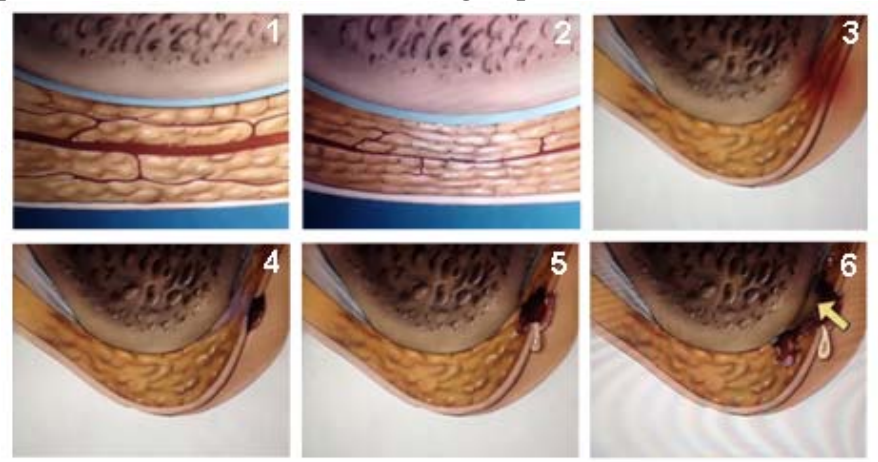

Fig. 1. Capillary occlusion - development of a pressure ulcer.

This project's main goal is to investigate the impact of extrinsic factors that contribute to the development of pressure ulcers, to identify critical pressure values in different anatomic points of risk and in addition to assess the impact of a specially designed support surface in preventing that the critical pressure values are reached. Through the incorporation of sensors, one intends to identify ranges of values of pressure, humidity and exposure times) corresponding to levels of comfort-discomfort and establish relationships between its magnitude and duration monitoring in the body's areas contacting with the support surfaces. The same principle used in this work can be applied to other situations, such as people with serious motor function limitations that condition their mobility and/or deambulation, bed rest patients or patients under effect of sedatives or anesthesia during long surgeries (intra and post operative).

and the motor actions, as taken by healthy users. These values will be used to define a protocol of sensor data acquisition versus warnings and actions (displayed on a monitor, through sound, or sending messages to a mobile phone) and/or to an intelligent automatic actuation system that will produce the same results that a healthy user achieves with changes in posture. The system is intended to enable the inversion of the trend of evolution of these values when critical limits are reached, acting automatically on the spot where risk conditions are being identified by the sensing surfaces.

This project is an innovative multidisciplinary undertaking, combining insights of textile, polymers, electronics, mechanics and psychological studies to develop support surface systems able to acquire, record and evaluate body pressure and humidity and react at limit values of discomfort with warnings and/or with surface actuation

\section{BACKGROUND}

Despite advances in medicine, pressure ulcers remain a major cause of morbidity and mortality. Pressure ulcers are areas of located necrosis of skin and subcutaneous tissues. They result of unrelieved pressure in a skin area, usually over a bony prominence, during a period of time enough to develop an erythema, in patients who remain immovable for long periods. These periods are not constant and vary according to several risk factors (Sensorial perception; Mobility and activity; tissue tolerance - related with extrinsic factors: humidity, friction and shear force and intrinsic factors: nutrition, age and arteriolar pressure; Aspects related to aging; Alterations of the body temperature; Degenerative chronic illnesses as diabetes and cancer). Pressure ulcers represent high costs in financial and human terms. In U.S.A. studies present estimated costs for treatment per ulcer between 200040000USD and 25000USD per patient for reconstruction surgery. Considering that elderly individuals become the 
fastest-growing segment of the population in the western population, and that they are in the risk factors, pressure ulcers will have an increasing economic impact. Prevention of pressure ulcers should be the main target in the management of this serious problem. Early identification of people in risk and timely adopted preventive strategies are cost effective measures and avoid adverse health consequences of pressure ulcers. Current data suggests that the use of special support surfaces may reduce pressure ulcers incidence in risk patient. This alternative product is widely used, as aids to prevention of pressure ulcers, in both institutional and non-institutional settings. Current National Institute for Health and Clinical Excellence guidelines on the prevention of pressure ulcers recommend the use of high specification foam mattresses as the standard in vulnerable patients and surfaces such as alternating pressure mattresses for high risk people. Several critical questions still remain unanswered: How much pressure and/or how long does it take to produce predictable ulceration? To what extent other extrinsic local factors, like temperature or humidity, contribute to skin damage? Is intermittent pressure relief effective in preventing critical pressure? Which characteristics should a support surface present to assure optimal skin conditions?

Pressure ulcers, also known as decubitus ulcers, pressure sores (or bedsores), are areas of localized damage to the skin and underlying tissue (graduated in stages I to IV)[1, 2] Pressure ulcers are commonly seen in the elderly and in patients with mobility limitations and impaired sensation that often are confined to bed. Trauma, burns, spinal cord injury, neurological deficits, peripheral vascular disease, stroke and faecal incontinence emerge as risk factors for pressure ulcers $[3,4]$

Pressure ulcers are common among patients hospitalized in acute- and chronic-care facilities. According to studies available, the incidence in hospitalized patients ranges from $4 \%$ to $29 \%$, and the prevalence in hospitalized patients is $3.5 \%$ to $69 \%$ [5-8] Actual knowledge suggests pressure ulcers result from constant pressure enough to impair local blood flow to soft tissue for an extended period. In view of the pressure ischemia theory, the external pressure must overcome the arterial capillary pressure of $32 \mathrm{~mm} \mathrm{Hg}$ to impair inflow and greater than venous capillary closing pressure of $8-12 \mathrm{~mm} \mathrm{Hg}$ to impede the return of flow for an extended time. Constant external pressure for 2 hours or more produces irreversible changes in tissues in animal model studies. The anatomic points of greatest pressure vary with the patient position. With the patient supine they include the sacrum, heel, and occiput, at $40-60 \mathrm{~mm} \mathrm{Hg}$. With the body in prone position, the chest and knees absorbed the greatest pressure, at $50 \mathrm{~mm} \mathrm{Hg}$. When the patient is sitting, the ischial tuberosities are under the most pressure, at $100 \mathrm{~mm} \mathrm{Hg}$ [9]

The common sites of pressure ulcers include the ischium $(28 \%)$, the sacrum $(17-27 \%)$, the trochanter $(12-19 \%)$, and the heel $(9-18 \%)[1,10,11]$. The geriatric and pediatric patients develop frequently ulcers in the occiput because they spend amounts of time lying supine. Pressure ulcers are costly in financial and human terms $[1,12,13]$.

The most important factor in the development of pressure ulcers is unrelieved pressure. Pressure ulcers arise from prolonged tissue ischemia caused by pressure that exceeds the tissue capillary pressure. Prolonged pressure deprives tissues of oxygen and essential nutrients, owing to ischemia and hypoxia, which then causes the ulcers [14].

Interface pressure, defined as "perpendicular force per unit area between the body and support surface"[1], is affected by the stiffness and composition of the body tissue and by the geometric shape of the body being supported. While interface pressures less than $32 \mathrm{~mm} \mathrm{Hg}$ are assumed by many clinicians to be safe, above $32 \mathrm{~mm} \mathrm{Hg}$ are thought to lead to closure of capillary beds and tissue ischemia. Products aimed at reducing or relieving pressure have tended to use interface pressure as the standard for judging product efficacy [15, 16]. Further investigation of the application of the criterion standard of $32 \mathrm{~mm} \mathrm{Hg}$ that resulted from Landis' work [17] to pressure ulcer pathology is needed, particularly since the transmission of load on tissue and muscle can decrease or increase based on characteristics of the tissue at different sites [18].

\section{STATE OF THE ART}

Relieving the pressure in the critical skin point may prevent the development of the ulcer. Current data suggests that special support surfaces, like pressure-relieving cushions, may reduce pressure ulcer incidence in patients at risk. This alternative product is widely used, as an aid to the prevention of pressure ulcers, in both institutional and non-institutional settings [19].

There are several proposals on the market advertising good results in prevention of pressure ulcers. Their costs are very high and effectiveness is far from satisfying the specialists [2025]

Studies carried out in clinical environment using special mattresses showed better effectiveness in the prevention of pressure ulcers than standard surgical surfaces, but require further investigation [26, 27-43].

Results of these studies indicated that the devices considered most efficient in prevention of skin injuries were, in decreasing order, the multi-cell pulsating dynamic mattress system, a dry viscous-elastic polymer pad and gel cushions. Nevertheless, they can bring benefits only to low risk patients and with normal skin [44].

We can classify these devices as follows: Air surfaces; Memory Foam surfaces; Adjustable surfaces; Latex surfaces; Water surfaces; innerspring surfaces.

None of the solutions available in the market provide permanent monitoring of the pressure exceeding the comfort limit. They only use sensor devices to monitor them in a PC as comparison between their solutions and standard products [2225]. Humidity or temperature values have never been measured.

To prevent the pressure ulcers risks, patients are moved periodically. Periods vary, as patients are different. In clinical 
environments, these periods can be based on scales of prediction of risk of pressure ulcers development - Norton, Gosnell, Knoll, Waterlow, Hemphil and Braden. The Braden scale is most used. It intends to evaluate the state of the patient in sub-scales (sensorial perception, mobility, activity, humidity, nutrition, and friction and shear). Nevertheless, studies show that all scales require a systematical evaluation and are only advised for internal patients. They are not used for surgical patients, bed rest patients or patients using wheelchairs [21].

\section{OBJECTIVES OF THE CURRENT PROJECT}

This project pursues several main objectives, all of them related to the prevention of the pressure ulcers, as described. The first objective is the development of a low-cost monitoring system that can be easily integrated into a mattress or cushion and provides continuous measurement of pressure, humidity and temperature.

Given these values, studies are to be carried out to determine their critical limits and their relation to exposure times and individual factors. This will make the development of warning systems possible.

The last objective is the development of actuation systems that compensate the critical factors determined by the monitoring system; these will be based on inflatable elements.

In this paper, we will describe the approach taken on the development of the pressure measurement system.

\section{GENERAL SYSTEM OVERVIEW}

The system is based on an array of capacitive sensors arranged in a matrix form (as described in the next section). A custom-designed, signal conditioning and acquisition board, controlled by a 8751 microcontroller, connects to a PC via the microcontroller's serial interface. A program developed in Labview reads, scales and displays the data.

\section{SENSOR DESIGN}

The pressure sensor is based on a parallel plate capacitor with polymeric foam between the plates. Fig. 2 shows the pressure $\mathrm{x}$ deformation curves obtained for 3 samples of the foam used in the sensor array fabrication.

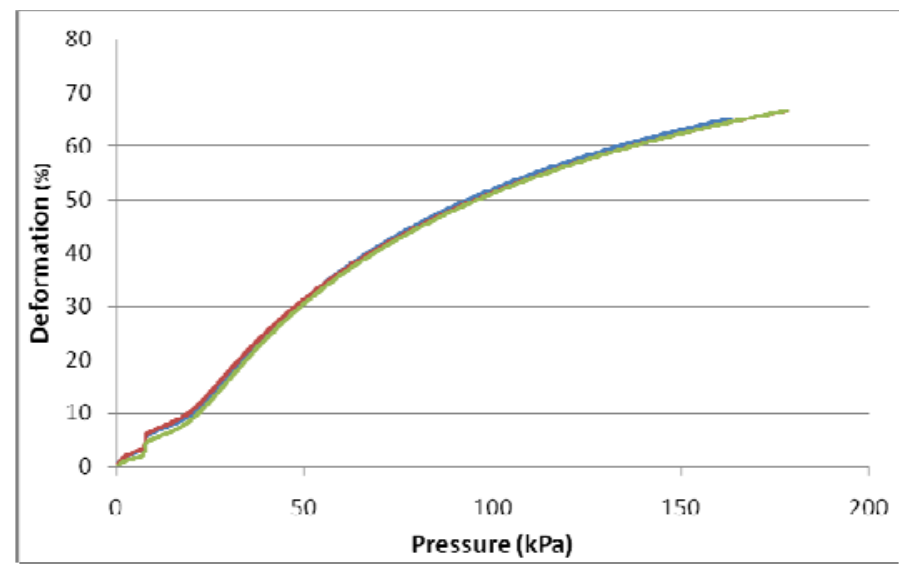

Fig. 2. Pressure $x$ deformation curves obtained for 6 samples of the rubber used in the sensor array fabrication carried out according to ASTM D 1621.

The curve can generally be very well approximated by a polynomial function. In the low-pressure range, the foam exhibits a quite irregular behavior. Considering that this is the least critical range in term of discomfort behavior, the resulting measurement error can be neglected and the calibration curve may be approximated by a polynomial function.

Fig. 3 shows a simple circuit for capacitance measurement. The current $i$ is given by $i=v \omega C$, with $v$ being the $\mathrm{RMS}$ value of applied voltage, $\omega$ the angular frequency of the voltage and $\mathrm{C}$ the capacitance. As $\mathrm{v}$ and $\omega=2 \pi f$ are constant, the value of $i$ is proportional to the capacitance $\mathrm{C}$.

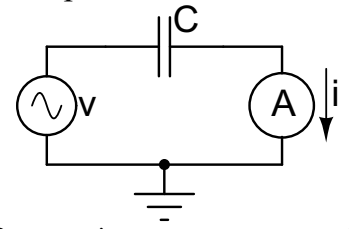

Fig. 3: Simple circuit for capacitance measurement: the current $i$ is given by $\mathrm{i}=\mathrm{v} \omega \mathrm{C}$. As $\mathrm{v}$ and $\omega=2 \pi \mathrm{f}$ are constant, the value of $\mathrm{i}$ is proportional to the capacitance $\mathrm{C}$.

Despite the simplicity of the proposed circuit there is a major drawback: the influence of the parasitic capacitances between the wires that connect the components. The same circuit is shown in Fig. 4, now considering the paristic capacitances. In this figure, $\mathrm{C}_{\mathrm{P} 1}$ represents the parasitic capacitance between the wire that connects the sensor to the voltage source and the ground; $\mathrm{C}_{\mathrm{P} 2}$ represents the parasitic capacitance between the wire that connects the sensor to the ammeter and the ground and $\mathrm{C}_{\mathrm{P} 3}$ represents the parasitic capacitance between the wire that connects the sensor to the voltage source and the wire that connects the sensor to the ammeter.

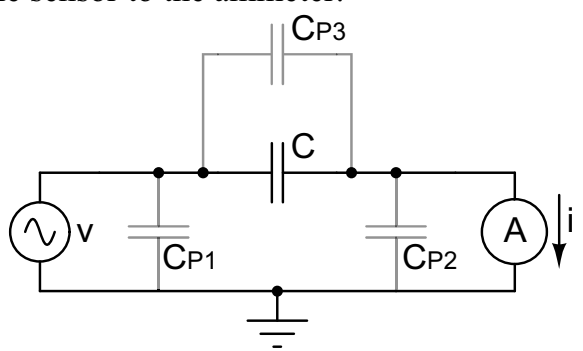

Fig. 4: Circuit of Fig. 3 with the parasitic capacitances between the wires represented.

It is important to note that the parasitic capacitance $\mathrm{C}_{\mathrm{P} 1}$ is placed parallel to the voltage source, so its value does not influence the measurement. On the other hand, $\mathrm{C}_{\mathrm{P} 2}$ is in parallel with the ammeter, which has very low impedance, so it is short-circuited and it also does not influence the measurement. The main problem is $\mathrm{C}_{\mathrm{P} 3}$, which is in parallel with the sensor capacitance and affects its measurement. In order to solve this problem, either the wire that connects the voltage source to the sensor, or the wire that connects the sensor to the ammeter (or both) must be shielded, with its shield connected to the ground. In this case, either the $C_{\mathrm{P} 1}$ value or $\mathrm{C}_{\mathrm{P} 2}$ value (or both values) increase, but, as seen before, they do not influence the measurements. 
In the present prototype, an array of $8 \times 8$ sensors was used, as it shown in Fig. 5. The bottom electrode is connected to the voltage source by means of a shielded wire. The top electrodes are connected to the readout circuit, which will be described in the next section.

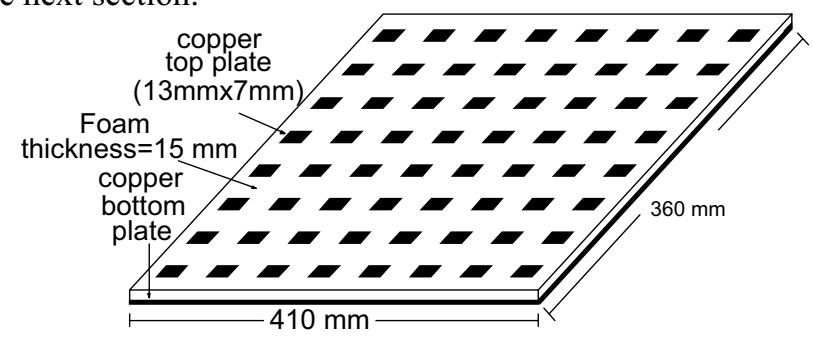

Fig. 5. Array of $8 \times 8$ capacitive sensors. The bottom electrode consists of a copper sheet with $410 \mathrm{~mm} \times 360 \mathrm{~mm}$; between the electrodes, a foam layer with $15 \mathrm{~mm}$ thickness was placed; the top electrodes consist in an array of $8 \times 8$ copper plates with $13 \mathrm{~mm} \times 7 \mathrm{~mm}$.

Fig. 6 shows a picture of the sensor array before its placement inside the cushion.

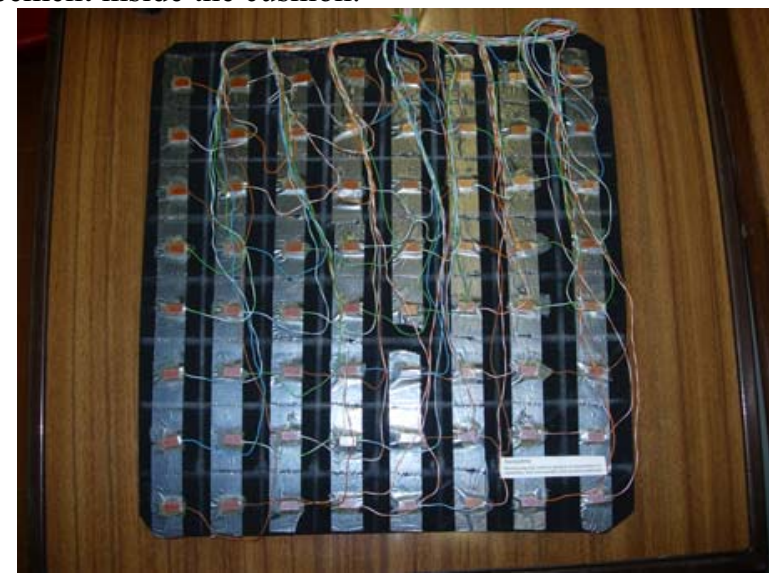

Fig. 6. Picture of the sensor array before its placement inside the cushion.

\section{CIRCUIT DESIGN}

\section{A. Voltage source}

As the voltage source, a Wien Bridge oscillator, working at the frequency of $40 \mathrm{kHz}$, has been used The output voltage is $10 \mathrm{~V}_{\mathrm{pp}}$. Fig. 7 shows the schematic diagram of the implemented circuit.

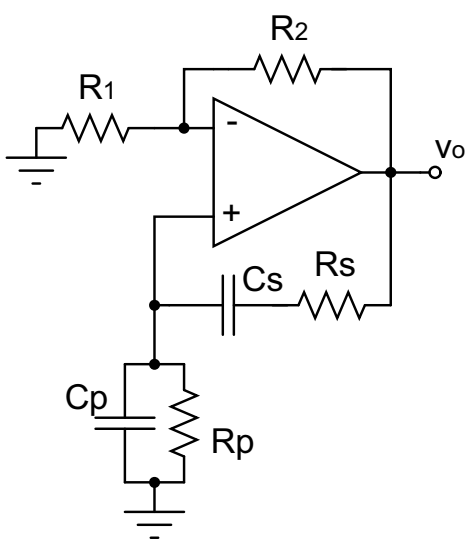

Fig. 7. Voltage source based on a Wien bridge oscillator.

\section{B. Readout circuit.}

In the sensor, the output current is proportional to the sensor's capacitance. When pressure is applied to the sensor, capacitance varies inversely proportional to the distance between the capacitor plates and thus proportionally to the applied pressure level.

In order to read the pressure level, it is necessary to convert the current supplied by the sensor into a voltage, since the data acquisition board works with voltages at its input. For that, it was necessary to implement the circuit of Fig. 8.

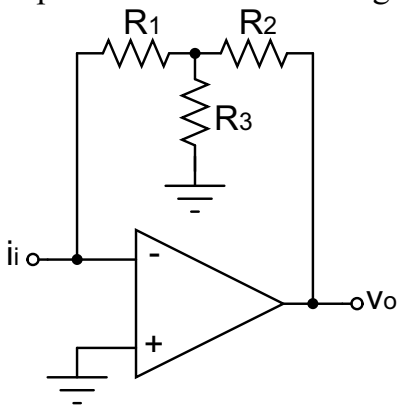

Fig. 8. Current-voltage converter.

The output of the circuit of Fig. 8, an AC voltage, most be rectified in order to be read by the data acquisition system. As we are dealing with small signals, it was be necessary to implement the circuit of Fig. 8: a precision rectifier for small input voltages.

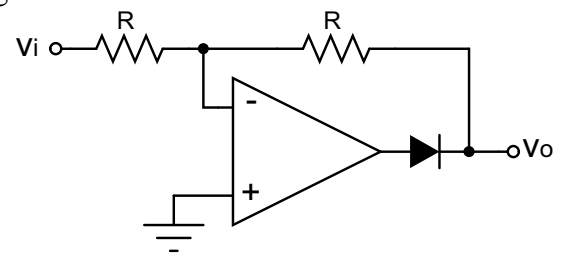

Fig. 8. Precision full-wave rectifier.

Finally, as the movement of the patients occurs at a low frequency, a first-order low-pass filter is used. The main goal of this filter is to eliminate the $80 \mathrm{kHz}$ frequency that comes from the full wave rectifier.

\section{SOFTWARE DESIGN}

The acquisition and display software is being developed in Labview. At the current stage of this work, it is a very simple program providing:

-serial communication with the data acquisition board, reading the acquired values from the board;

-scaling of the binary values according to pre-defined calibration curves;

-display of the values on a $2 \mathrm{D}$ graph in which the pressure values are mapped to a color scale;

Additionally, there is a calibration function that allows the application of varying pressure values on the sensors and the readout of the resulting voltages. This is done by placing a metal plate over the sensor matrix and applying known force values. Calibration curves for each sensor in the matrix are computed in the process. 
IX.

EXPERIMENTAL PROTOTYPE AND RESULTS

Since there are currently only 64 sensors, a clearer display is achieved by interpolating the values in the matrix, as shown in Fig. 9 and 10. An alternative display is a 3D surface graph.

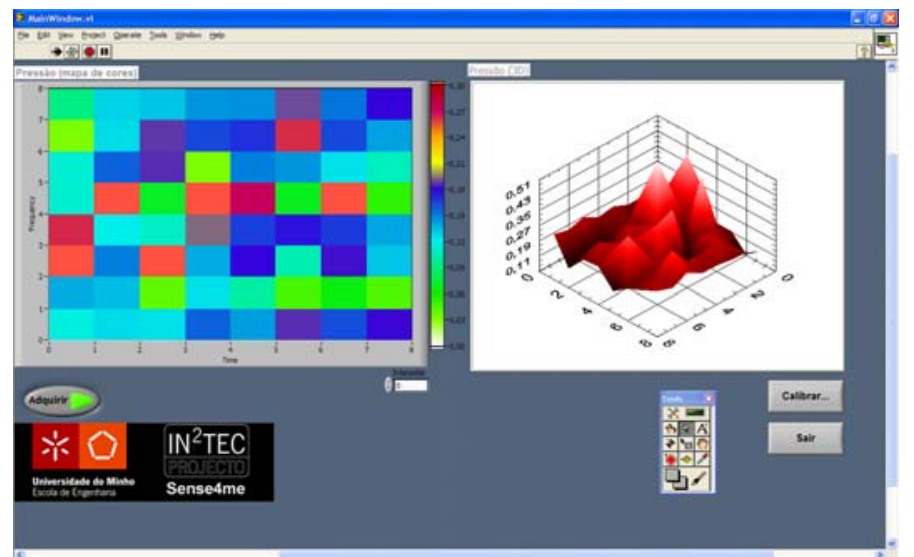

Fig. 9. Screenshot of the software. Subject is sitting on a wheelchair with sensor matrix. Interpolation disabled in the 2D-colour graph

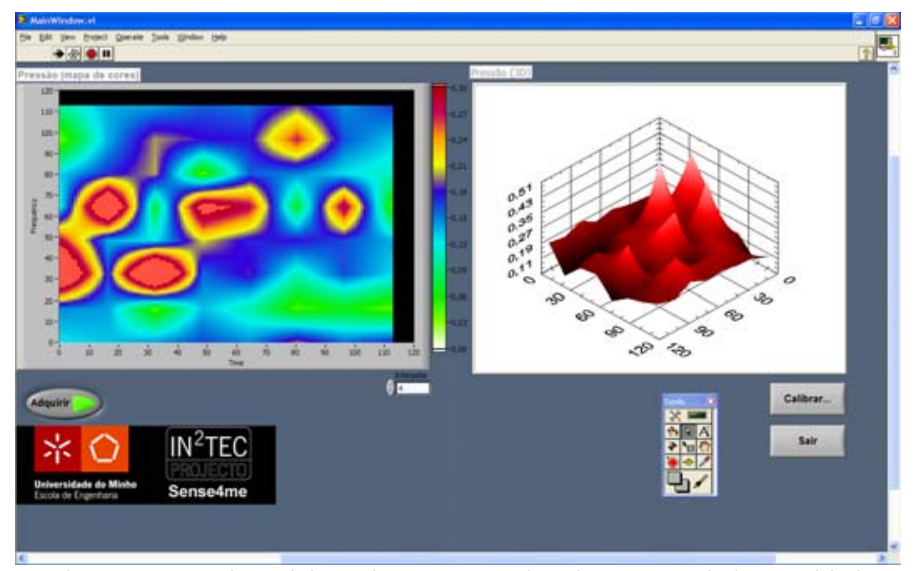

Fig. 10. Screenshot of the software, same situation, Interpolation enabled.

Fig. 11 shows a picture of the readout electronics board. For this first prototype, this board is composed by 64 channels.

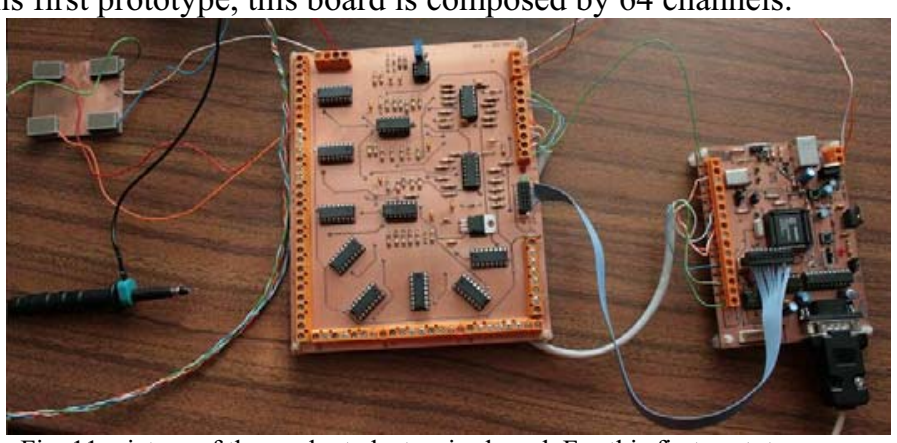

Fig. 11. picture of the readout electronics board. For this first prototype

Fig. 12 shows the developed prototype of the wheelchair with the cushion equipped with the sensor matrix and the monitoring system.

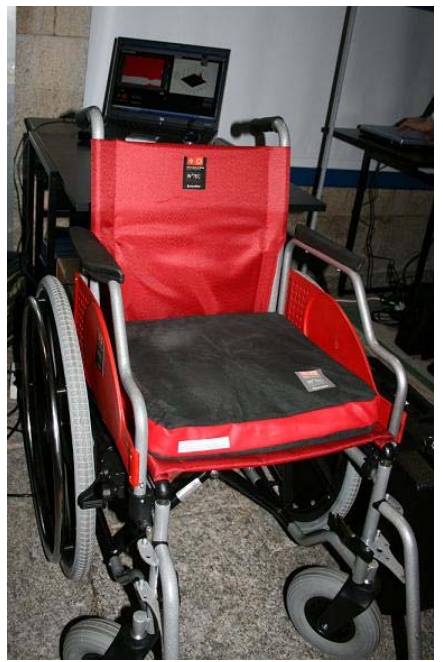

Fig. 12. First prototype - Cushion for wheelchair with sensor matrix and monitoring system.

\section{CONCLUSIONS}

This article described the design, fabrication and experimental results of a pressure sensor array embedded in a cushion and its readout electronics. The pressure sensor array is based on capacitive sensors whose dielectric is constituted by polymeric foam. As this is the first prototype, the obtained results are very promising and encouraging. Nevertheless, there are some issues that must be improved in the next prototypes. The first aspect is the calibration, which should be made easier by allowing the calibration of a single sensor and the use of the resulting calibration parameters for the remaining sensors. The application of a controlled force to the whole sensor array is much more difficult. This can only be achieved by a better control of the dimensional and physical parameters of the sensor fabrication.

Another aspect is the elimination of the rigid copper electrodes. These can cause discomfort to the patient sitting on the wheelchair. In the current prototype, a gel cushion is inserted between the measuring cushion and the patient, but this cushion will somewhat distort the measured force values. Experiments are being conducted to replace the copper plates by textile fabric produced with inserted stainless steel yarns. These metallic yarns will be used as the plates of the capacitive sensors, providing an all-textile, flexible and soft sensor array. The approach has already been validated, a prototype is expected to be demonstrated on short-term.

\section{ACKNOWLEDGEMENTS}

The authors thank the Portuguese Foundation for Science and Technology - FCT (Grant PTDC/SAU-BEB/68678/2006) and the School of Engineering of The University of Minho (IN ${ }^{2}$ TEC project). 


\section{REFERENCES}

[1] National Pressure Ulcer Advisory Panel. Pressure ulcers: incidence, economics, risk assessment 1989

[2] Calibri, MHL A utilização da pesquisa na prática clínica da enfermagem: limites e possibilidades 2002

[3] Reed, R Low serum albumin levels, confusion, and fecal incontinence: are these risk factors for pressure ulcers in mobility impaired hospitalized adults? 2003

[4] Allman R A Pressure ulcer risk factors among hospitalized patients with activity limitation 1995

[5] Hickerson, WL Comparison of total body tissue interface pressure of specialized pressure-relieving mattresses 2004

[6] Berlowitz, D Risk factors for pressure sores. A comparison of crosssectional and cohort-derived data 1989

[7] Meehan, M Multisite pressure ulcer prevalence survey 1990

[8] Shannon, M Pressure ulcer prevalence in two general hospitals 1989

[9] Lindan, O Pressure distribution on the surface of the human body. Evaluation in lying and sitting positions using a bed of springs and nails 1965

[10] Allman, R Pressure ulcers among the elderly 1998

[11] Fuhrer, M Pressure ulcers in community-resident persons with spinal cord injury: prevalence and risk factors 1993

[12] Bergstrom, N; Braden BA prospective study of pressure sore risk among institutionalized elderly 1992

[13] Bergstrom, N Treatment of Pressure Ulcers 1994

[14] Kosiak, M Prevention and rehabilitation of pressure ulcers 1991

[15] Krouskop, TA The effectiveness of preventive management in reducing the occurrence of pressure sores 1983

[16] Salcido, R A reliable animal model of pressure sore development: The role of free radicals 1993

[17] Lindan, O Etiology of decubitus ulcers. An experimental study 1961

[18] Bennett, L Pressure versus shear in pressure sore causation 1985

[19] National Institute for Health and Clinical Excellence. Pressure ulcer prevention: pressure ulcer risk assessment and prevention, including the use of pressure-relieving devices (beds, mattresses and overlays) for the prevention of pressure ulcers in primary and secondary care 2003

[20] Barbara, P Introducing the pressure support surfaces from Kaymed 2001

[21] Costa, IG Incidence of pressure ulcer and risk factors in patients of an Intensive Care Center 2003

[22] www.tempurpedic.com

[23] www.kaymedworld.com

[24] www.satbed.com
[25] www.tekscan.com

[26] Ursi, ES Perioperative prevention of skin injury: an integrative literature review 2005

[27] Kleinbeck, SVM Developing nursing diagnosis for a perioperative care plan 1989

[28] Rossi, LA Diagnósticos de enfermagem do paciente cirúrgico no período pós-operatóro imediato 2000

[29] Piccoli, M Enfermagem perioperatória: identificação dos diagnósticos de enfermagem na visita pré-operatória fundamentada no modelo conceitual de Levine 2004

[30] Aranovitch, SA A comparative study of an alternating air mattress for the prevention of pressure ulcers in surgical patients 1999

[31] Russel, JA Randomized controled trail to determine the safety and efficacy of multicell pulsanting dynamic mattress system in the prevention of pressure ulcers in patients undergoing cardiovascular surgery 2000

[32] Nixon, J A sequencial randomized controled trail comparing a dry viscoelastic polymerpad and standard operating table mattress in the prevention of post-operative pressure sores 1998

[33] Scott, EM Effects of warming therapy on pressure ulcers - a randomized trial 2001

[34] Schultz, A Etiology and incidence of pressure ulcers in surgical patients 1999

[35] Scott, EM Measurement of interface pressures in the evaluation of operating theatre mattresses 1999

[36] Blaylock, B et al Measuring tissue interface pressures of two support surfaces used in the operating room 1994

[37] Cullum, N Beds, mattresses and cushions for pressure sore prevention and treatment 2004

[38] Defloor, T Do pressure relief cushions really relieve pressure? 2000.

[39] Defloor, T Preventing pressure ulcers: an evaluation of four operatingtable mattresses 2000

[40] Hawkins, JE The effectiveness of pressure reducing table pads as an intervention to reduce the risk of intra-operatively acquired pressure sensors. 1997

[41] Hoshoski, VM Intraoperative pressure sore prevention: an analysis of bedding materials 1994

[42] Chalian, AA Backside first in head and neck surgery?: preventing pressure ulcers in extended length surgeries 2001

[43] Armstrong, D An integrative review of pressure relief in surgical patients 2001

[44] Andrychuck, MA Pressure ulcers: causes, risk factors, assestment, and intervention 1998 